\title{
On the role of invariants for the parameter estimation problem in Hamiltonian systems
}

\author{
M. Baake \\ Institut für Theoretische Physik, Universität Tübingen, Auf der Morgenstelle 14, 72076 Tübingen, Germany \\ E. Baake \\ Max-Planck-Institut für Entwicklungsbiologie, Spemannstrasse 35/IV, 72076 Tübingen, Germany \\ and \\ E. Eich \\ Linde AG, Werksgruppe VA, Dr.-Carl-von-Linde-Strasse, 82049 Höllriegelskreuth, Germany
}

Received 28 July 1992; revised manuscript received 30 April 1993; accepted for publication 14 June 1993 Communicated by A.P. Fordy

\begin{abstract}
The parameter estimation problem is discussed for differential equations that describe a Hamiltonian system. Since the conserved total energy is an invariant which contains all parameters of the system, we can achieve parameter estimation without any numerical integration. This is demonstrated for data in the chaotic region of the Hénon-Heiles system and of the planar double pendulum. We show that the method works well for ideal as well as noisy data. In this context, an appropriate method for the generation of reliable time series in the presence of an invariant is discussed. Finally, it is shown that our method also provides a simple approach to global fitting in discrete dynamical systems with invariants.
\end{abstract}

\section{Introduction to problem and method}

It has recently been demonstrated that fitting an ordinary differential equation (ODE) to chaotic data may be a well-posed problem when treated with boundary value problem methods in a suitable manner [1]. The method was illustrated with examples from the class of dissipative systems. Nevertheless, one would expect the method to work for Hamiltonian systems as well, compare ref. [2] for an example.

On the other hand, conservative Hamiltonian systems are quite different from dissipative systems: they possess the total energy as a global invariant, i.e., the dynamics is restricted to a manifold of reduced dimension. This immediately suggests an alternative procedure for parameter estimation which we will describe after a short explanation of the estimation problem as such.

Consider the following situation that commonly arises in dealing with nonlinear phenomena. On the one hand, experimental time series are obtained from measurements; on the other hand, an ODE model of the underlying process is formulated which usually contains as yet undetermined parameters. In order to judge this model, one has to find the set of parameters for which the model equations describe the measured dynamics best. If a good fit is obtained, the newly found knowledge of the parameters will allow predictions from the model; if there are substantial deviations, these may give clues concerning the necessary refinements of the model. Methods to tackle this problem of parameter estimation in ODEs have been described in ref. [3], and have been applied to the particularly challenging chaotic case in ref. [1]. In that paper, the authors started from the following three key assumptions: 
(A1) The dimension of the ODE system is either known or reliably guessed.

(A2) The model or the model class to describe the data is specified.

(A3) The sampling rate of the data is sufficiently high with respect to all relevant frequencies occurring.

Let us now assume that our time series $\mathscr{S}$ of phase space points measured at instants $t_{i}, \mathscr{S}=\left\{\left(\boldsymbol{p}_{i}, \boldsymbol{q}_{i}\right) \mid i=\right.$ $1, \ldots, N\}$, is described by a Hamiltonian system which is not completely integrable in order to exclude the simpler situation of fitting explicit orbits to data. The Hamiltonian $H(p, q ; \gamma)$ is taken to be independent of time ${ }^{\# 1}$, but dependent on parameters $\gamma$ (such as masses, frequencies, lengths, or interacting strengths) which are to be determined. That is, the following optimization problem (with $H$ of dimension $n$ ) is to be solved,

$F_{1}(\mathscr{S} ; \gamma)=\sum_{i=1}^{N} \sum_{j=1}^{n}\left[\left(\frac{p_{i j}-p_{j}\left(t_{i}, \gamma\right)}{\sigma_{p_{i j}}}\right)^{2}+\left(\frac{q_{i j}-q_{j}\left(t_{i}, \gamma\right)}{\sigma_{q_{i j}}}\right)^{2}\right] \stackrel{!}{=} \min _{\gamma}$

such that

$\dot{q}=\frac{\partial H(p, q ; \gamma)}{\partial p}, \quad \dot{p}=-\frac{\partial H(p, q ; \gamma)}{\partial q}$.

Here, $p_{i j}, q_{i j}$ and $p_{j}\left(t_{i}, \gamma\right), q_{j}\left(t_{i}, \gamma\right)$ denote the measured values of component $j$ and the solution of the corresponding differential equations with parameters $\gamma$, respectively, and the symbol $\stackrel{!}{=}$ in (1) indicates that the left hand side is to be minimized with respect to $\gamma$. If the measurement errors are mutually independent and normally distributed with zero mean and standard deviations $\sigma_{p i j}, \sigma_{q i j}$, the solution of (1), (2) gives a maximum likelihood estimate of $\gamma$.

If assumptions (A1)-(A3) are fulfilled, this solution can be found with the BVP approach described in ref. [1], which is obtained by discretization of (1), (2). This has actually been applied to the Hénon-Heiles system in ref. [2]. It will, however, fail if assumption (A3) is violated, for numerical integration must run into problems in the case of low sampling rates - at least in the chaotic case.

We therefore observe that, if full phase space information is available (either directly or by suitable reconstruction), the existence of an invariant set or manifold

$\mathscr{M}_{E}=\left\{(\boldsymbol{p}, \boldsymbol{q}) \in \mathbb{R}^{2 n} \mid H(\boldsymbol{p}, \boldsymbol{q} ; \gamma)=E\right\}$

allows us to reduce the dynamical fitting task (1), (2) to the statical problem of fitting a surface to the data points, which is a standard problem of constrained optimization [4],

$F_{2}\left(\mathscr{S}, \tilde{P}_{;} \gamma\right)=\sum_{i=1}^{N} \sum_{j=1}^{n}\left[\left(\frac{p_{i j}-\tilde{p}_{i j}}{\sigma_{p_{i j}}}\right)^{2}+\left(\frac{q_{i j}-\tilde{q}_{i j}}{\sigma_{q i j}}\right)^{2}\right] \stackrel{!}{=} \min _{\tilde{\mathscr{I}}, \gamma}$

such that

$H\left(\tilde{\boldsymbol{p}}_{i+1}, \tilde{\boldsymbol{q}}_{i+1} ; \gamma\right)=H\left(\tilde{\boldsymbol{p}}_{i}, \tilde{\boldsymbol{q}}_{i} ; \gamma\right), \quad i=1, \ldots, N-1$.

Here, $\tilde{\mathscr{S}}=\left\{\tilde{\boldsymbol{p}}_{i}, \tilde{\boldsymbol{q}}_{i} \mid i=1, \ldots, N\right\}$ denotes the orbit of phase space points of the model physical process, wherefore they lie on the invariant set exactly - in contrast to the measured data points. This way, one can, of course, only determine parameters which show up in the invariant. However, the emergence of the differential equations (2) from the Hamiltonian $H(\boldsymbol{p}, \boldsymbol{q} ; \gamma)$ implies that all parameters do occur in the invariant and that the approach (4), (5) is reasonable, although this has, to our knowledge, not been used to solve the dynamical problem so far.

Nevertheless, we can reduce the problem even one step further. With wrong parameters $\gamma$ (e.g., rough estimates) our points will not lie on a single manifold $\mathscr{M}_{E}$, wherefore the expression $\sum_{i<j}\left[H\left(\boldsymbol{p}_{i}, \boldsymbol{q}_{i} ; \gamma\right)-H\left(\boldsymbol{p}_{j}, \boldsymbol{q}_{j} ; \gamma\right)\right]^{2}$ for a series $\mathscr{S}$ of phase space points will not vanish. But this expression is

\#1 The non-autonomous case can be made autonomous by the standard enlargement of phase space. 
- up to a factor $1 / N^{2}-$ the variance of the energy time series. We therefore define

$F_{3}(\mathscr{P} ; \gamma)=\frac{1}{N^{2}} \sum_{i<j}^{N}\left[H\left(\boldsymbol{p}_{i}, \boldsymbol{q}_{i} ; \gamma\right)-H\left(\boldsymbol{p}_{j}, \boldsymbol{q}_{j} ; \gamma\right)\right]^{2}$,

which has the advantage that $F_{3}$ is normalized per point and allows direct comparison of calculations for different numbers $N$ of points in phase space ${ }^{\# 2}$. Our approach then reduces to the simple unconstrained least squares minimization

$F_{3}(\mathscr{S} ; \gamma) \stackrel{!}{=} \min _{\gamma}$

where $F_{3}$ is calculated on a given, finite orbit in phase space. This approach is slightly more general (and, hence, weaker) than the previous one because one only needs some statistical properties of the time series which could be advantageous for more complicated invariant sets than (3). A careful analysis of this objective function (or variants thereof with higher moments) is certainly an interesting question, but not our concern here.

The most striking property of the approach (4), (5) or (7) is that one avoids any numerical integration. We will show by three examples that this works exceptionally well for ideal data (i.e., noise-free ones) and still quite satisfactorily for noisy data. Apart from a large computational advantage (no additional initial value problems need to be solved for the computation of the sensitivity matrices required in the optimization process), this makes possible the treatment of arbitrarily low sampling rates - or arbitrarily long time series, which can also cause problems in the chaotic case [1]. We will also tackle the case where missing components have to be reconstructed from incomplete data - which, of course, reintroduces dependence on (A3).

\section{Generation of data points}

Before one can test these ideas practically, it is necessary to think about adequate generation of data points - ideal as well as noisy ones. We have to circumvent problems caused by systematic deviations of numerical time series from the invariants, because otherwise we could violate assumption (A2) in an uncontrolled way by leaving the specified model class. Therefore, let us briefly describe a suitable approach to integrate the ODE

$\dot{y}=f(y)$,

where the solution $\boldsymbol{y}$ fulfills the invariant

$\boldsymbol{\theta}(\boldsymbol{y} ; \gamma)=0$.

This contains Hamiltonian systems via $\boldsymbol{y}=(\boldsymbol{p}, \boldsymbol{q})^{\mathrm{t}}$ and eq. (9) covers the case of an invariant by $\theta(\boldsymbol{y} ; \gamma)=$ $H(\boldsymbol{p}, \boldsymbol{q}, \gamma)-E$. Even if we are only interested in Hamiltonian systems here, we should mention that the brief explanations of this section apply to the more general class of differential-algebraic equations [5].

Numerical discretization schemes for the ordinary differential equation (8) are, in general, not adapted to preserve the invariant (9). The solution at point $t_{n}, \boldsymbol{y}\left(t_{n}\right)$, is approximated by $\boldsymbol{y}_{n}$ which is computed by a discretization scheme. In the case of one-step methods one obtains

$y_{n}=y_{n-1}+h \Phi\left(y_{n-1}, h\right)$,

where $h$ is the step size and $\Phi$ describes the discretization scheme, e.g., $\Phi=f\left(y_{n-1}\right)$ in the explicit Euler method. In the case of linear multistep methods, $y_{n}$ is calculated as the solution of

\#2 It is of no importance here to distinguish between $N$-weighted and $(N-1)$-weighted estimates for the variance - the minimizing set of parameters is the same. 
$\sum_{i=0}^{k} \alpha_{i} y_{n-i}+h \sum_{i=0}^{k} \beta_{i} f\left(y_{n-i}\right)=0$,

with suitably chosen coefficients $\alpha_{i}, \beta_{i}$ of the multistep method, compare ref. [6] for a discussion of several algorithms in the context of dynamical systems and chaos. Such a discretization scheme generally does not obey the invariant (9), i.e. we have $\Theta\left(y_{n} ; \gamma\right) \neq 0$. In addition, due to error propagation, this leads to a drift-off from the invariant and yields physically meaningless solutions.

To overcome this difficulty, specially adapted projection methods have been developed [5,7-9]. Let us mention that symplectic integrators, compare ref. [10], are no alternative as they neither conserve energy [11] nor increase precision in general [12]. We therefore focus on the projection method of refs. [7,9], which consists of two steps:

(1) The solution $\hat{\boldsymbol{y}}_{n}$ of the discretization scheme is computed. This computation is based on projected values in the last time steps.

(2) The solution is projected back onto the manifold given by the invariant, i.e., the final solution $y_{n}$ is computed (e.g., by Gauss-Newton methods) as the solution of

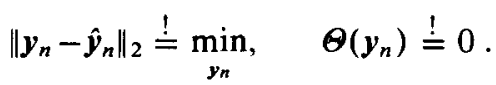

These values are then used to advance the solution.

This method has been proposed for the case of one-step methods [7] and for the case of multistep methods of the BDF type (backward difference formula) [9]. We would like to mention that the convergence of these projection methods has been shown [5] and that, in addition, the techniques used to prove convergence in ref. [9] actually allow insight into the remaining kind of error propagation: it can be shown that only the projected errors are propagated which is the reason for the higher accuracy of the solution in comparison with the standard methods mentioned earlier.

With this projection method in hand, we are able to calculate ideal data points in a reliable way and can then add noise to them. In this article, noise is always Gaussian with zero mean. The standard deviation specified is absolute and given as a fraction of the average modulus of the component considered, i.e., the origin of noise is assumed to be independent of the system dynamics ${ }^{\sharp 3}$. We will now illustrate our approach by means of two classical Hamiltonian systems and one discrete dynamical system.

\section{The Hénon-Heiles system}

Let us first consider the well-known Hénon-Heiles system $[14,15]$. With $\boldsymbol{p}=\left(p_{1}, p_{2}\right)^{\mathrm{t}}$ and $\boldsymbol{q}=\left(q_{1}, q_{2}\right)^{\mathrm{t}}$, the Hamiltonian reads

$H(\boldsymbol{p}, \boldsymbol{q})=\frac{1}{2}\left(p_{1}^{2}+p_{2}^{2}+q_{1}^{2}+q_{2}^{2}\right)+q_{1}^{2} q_{2}-\frac{1}{3} q_{2}^{3}$

and one obtains the equations

$\dot{q}_{i}=p_{i}, \quad i=1,2$,

$\dot{p}_{1}=-\left(q_{1}+2 q_{1} q_{2}\right), \quad \dot{p}_{2}=-\left(q_{2}+q_{1}^{2}-q_{2}^{2}\right)$.

This system is known to behave chaotically for $E>\frac{1}{9}$, compare refs. [14,15]. We now try to identify this system from the more general class of Hamiltonians given by

*3 The relative noise often used is easier to handle, compare ref. [13] and references therein, but the assumption of noise proportional to the individual signal amplitude is rather artificial on physical grounds. 
$H(\boldsymbol{p}, \boldsymbol{q})=\frac{p_{1}^{2}}{2 m_{1}}+\frac{p_{2}^{2}}{2 m_{2}}+\frac{\omega_{1}^{2}}{2} q_{1}^{2}+\frac{\omega_{2}^{2}}{2} q_{2}^{2}+W\left(q_{1}, q_{2}\right)$,

where $W\left(q_{1}, q_{2}\right)$ contains cubic terms (and thus gives rise to nonharmonic interactions),

$$
W\left(q_{1}, q_{2}\right)=a q_{1}^{3}+b q_{1}^{2} q_{2}+c q_{1} q_{2}^{2}+d q_{2}^{3} \text {. }
$$

Note that due to the coupling between the oscillators we do not have the freedom to renormalize masses and frequencies independently. Their values have to be determined from the measured data set.

For our numerical tests, we have generated a finite orbit of (13) (i.e., simulated data) with the method of section 2 . We chose our initial conditions in the chaotic region, namely $\left(p_{1}, p_{2}, q_{1}, q_{2}\right)_{0}=(0.4,-0.4,0.0,0.0)$, and sampled 200 data points with equidistant time intervals of $\Delta t=1.0$ (which yields some seven to eight data points per oscillation on an average ). Note that the parameters are implicitly fixed by the form of (12), i.e., $m_{1}=m_{2}=\omega_{1}=\omega_{2}=1$ and $a=c=0, b=1$, and $d=-\frac{1}{3}$ when compared with (14) and (15).

Hamiltonian (14) is invariant under a sign change of $\omega_{1}$ or $\omega_{2}$, wherefore we restricted the search to the physical region $\omega_{k} \geqslant 0, k=1,2$. Futhermore, if a specific Hamiltonian $H$ solves our minimization problem (7), any multiple of it is also a solution. This reflects the freedom to choose the units of measurement. Therefore, one has to fix one parameter in (14), e.g., $m_{1}=1$. Doing so, we remain with seven parameters to be determined. To apply our fitting approach via $F_{3}$, we only needed a very simple Monte Carlo algorithm that uses the method of successive annealing. In one iteration loop of our program, the Monte Carlo variations of the parameters are confined to a finite interval of the form $\left[2^{-n-1}, 2^{-n}\right]$ multiplied by the (fixed) initial step size. If for a certain number of trials no improvement (i.e., decrease of objective function $F$ ) is found, $n$ is replaced by $n+1$ until a given iteration depth is reached ${ }^{\sharp 4}$. The intial step size for the different parameters can of course be controlled externally. For more information on this type of algorithms, we refer to ref. [16]. Since parameter dependence of the present example is linear, the latter can easily be reformulated as a linear problem and then treated by QR decomposition as well [17], which we will comment on later. However, our next example will be more complicated, and we will apply different methods there.

Let us describe the results. We do not present a table because there is no particular value in the numbers, but only in the typical behaviour. For ideal data, i.e., the noise-free ones, we got all seven parameters right with accuracy of at least six digits, independently of the initial guesses. It did not matter whether we used 20 , 50 , or 100 data points, and $F_{3}$ was always of the order $10^{-15}$. The same result was obtained taking every tenth point of our time series (which simulates low sampling rates) as well as taking a random selection of them (which simulates a total loss of the time information). But this result is not too astonishing in view of perfectness and completeness of data.

Let us continue with complete but noisy data. Gaussian random numbers with zero mean were added to the data points, standard deviation was $1 \%,(5 \%)$ of the average modulus, respectively, calculated separately for each component (compare section 2). We used $50(100)$ points to obtain, $m_{2}, \omega_{1}, \omega_{2}$ with accuracy better than $1 \%,(5 \%)$ and $b, d$ with accuracy better than $3 \%,(20 \%)$. Parameters $a, c$ had modulus $<3 \times 10^{-3}$, $\left(1 \times 10^{-2}\right)$ which allows unambiguous identification of the right Hamiltonian. One gets essentially the same result by means of the standard $Q R$ decomposition technique mentioned above $\#$. This indicates an amazing amount of robustness, in particular in view of the fact that, for noisy data, $F_{3}$ of $(6)$ is almost certainly not the optimal objective function.

Finally, let us try to tackle incomplete data, e.g., given positions $q_{i}$ but missing momenta $p_{i}=m_{i} \dot{q}_{i}$. One can reconstruct them by numerical differentiation - but then, to keep approximation errors small, we need key assumption (A3). The latter is fulfilled for our time series. Using a second (fourth) order difference formula and 100 points we found $m_{2}, \omega_{1}, \omega_{2}$ with accuracy of $5 \ldots 15 \%(2 \ldots 5 \%)$ and $b, d$ with $15 \ldots 30 \%(10 \ldots 15 \%)$, while

\#4 This simple program was developed more than ten years ago together with B. Gemünden.

$\#$ We are grateful to K.M. Briggs for performing a series of calculations along these lines. 
$|a|,|c|$ were below $2.5 \times 10^{-2}\left(2 \times 10^{-2}\right)$ and $F_{3}$ was around $7 \times 10^{-5}\left(4 \times 10^{-5}\right)$. This could be improved considerably by restricting the model class, e.g., by imposing $a=c=0$, but we will drop the details. Instead, let us add Gaussian noise with $1 \%$ standard deviation to the data (before the reconstruction by the fourth order formula ). The result was $m_{2}, \omega_{1}, \omega_{2}$ with an accuracy of $2 \ldots 4 \%, b, d$ with an accuracy of $12 \ldots 18 \%$, whereas $|a|$, $|c|$ were below $3 \times 10^{-2}$ and $F_{3}$ around $4 \times 10^{-5}$. Let us now turn to a more complicated example where the parameter dependence is nonlinear.

\section{The planar double pendulum}

In this second example, we will use the Lagrangian formulation because it leads to slightly simpler expressions in the ODEs and because it might be instructive to see explicitly that we do not depend on a specific presentation of the system. Consider the Lagrangian $[18,19] L=T-V$ with

$T=\frac{1}{2}\left(m_{1}+m_{2}\right) l_{1}^{2} \dot{\varphi}_{1}^{2}+\frac{1}{2} m_{2} l_{2}^{2} \dot{\varphi}_{2}^{2}+m_{2} l_{1} l_{2} \dot{\varphi}_{1} \dot{\varphi}_{2} \cos \left(\varphi_{1}-\varphi_{2}\right)$

and

$V=-\left(m_{1}+m_{2}\right) g l_{1} \cos \left(\varphi_{1}\right)-m_{2} g l_{2} \cos \left(\varphi_{2}\right)$

for the planar double pendulum of fig. 1 .

Via the Euler-Lagrange equations

$\frac{\partial L}{\partial \varphi_{i}}-\frac{\mathrm{d}}{\mathrm{d} t} \frac{\partial L}{\partial \dot{\varphi}_{i}}=0, \quad i=1,2$,

one derives the second order equations $\# 6$

$\ddot{\varphi}_{1}=\frac{1}{\mu-\cos ^{2}\left(\varphi_{1}-\varphi_{2}\right)}\left|\begin{array}{cc}-\sin \left(\varphi_{1}-\varphi_{2}\right) \dot{\varphi}_{2}^{2}-\mu \lambda \omega_{1}^{2} \sin \left(\varphi_{1}\right) & \cos \left(\varphi_{1}-\varphi_{2}\right) \\ \sin \left(\varphi_{1}-\varphi_{2}\right) \dot{\varphi}_{1}^{2}-\omega_{1}^{2} \sin \left(\varphi_{2}\right) & 1 / \lambda\end{array}\right|$

and

*6 By means of the standard substitution $\psi_{i}=\dot{\varphi}_{i}, i=1,2$, one obtains the set of first order equations needed for the numerical integration along the lines of section 2 .

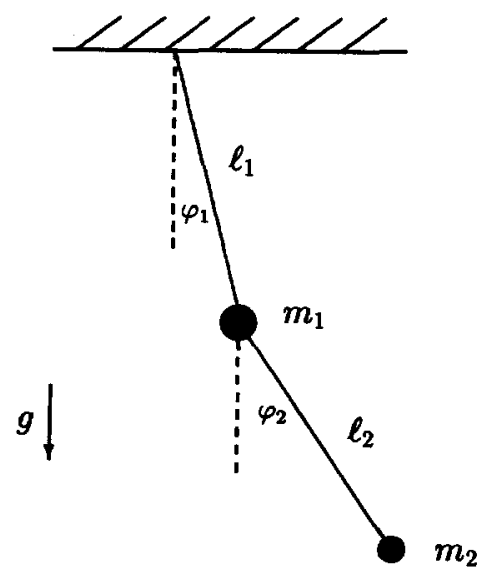

Fig. 1. The planar double pendulum. 


$$
\ddot{\varphi}_{2}=\frac{1}{\mu-\cos ^{2}\left(\varphi_{1}-\varphi_{2}\right)}\left|\begin{array}{cc}
\mu \lambda & -\sin \left(\varphi_{1}-\varphi_{2}\right) \dot{\varphi}_{2}^{2}-\mu \lambda \omega_{1}^{2} \sin \left(\varphi_{1}\right) \\
\cos \left(\varphi_{1}-\varphi_{2}\right) & \sin \left(\varphi_{1}-\varphi_{2}\right) \dot{\varphi}_{1}^{2}-\omega_{1}^{2} \sin \left(\varphi_{2}\right)
\end{array}\right|
$$

where we introduced $\omega_{1}=\sqrt{g / l_{1}}, \lambda=l_{1} / l_{2}$ and $\mu=1+m_{1} / m_{2}$. To exclude trivial cases, we assume $0<\lambda<\infty$ and $1<\mu<\infty$ in what follows, i.e., a true double pendulum. This also ensures that the denominator in (18) and (19) never vanishes. The conserved total energy reads

$$
\begin{aligned}
& E\left(\dot{\varphi}_{1}, \dot{\varphi}_{2}, \varphi_{1}, \varphi_{2} ; \omega_{1}, \lambda, \mu\right)=T+V \\
& \quad=m_{2} l_{1} l_{2}\left\{\frac{1}{2} \mu \lambda \dot{\varphi}_{1}^{2}+(1 / 2 \lambda) \dot{\varphi}_{2}^{2}+\dot{\varphi}_{1} \dot{\varphi}_{2} \cos \left(\varphi_{1}-\varphi_{2}\right)-\omega_{1}^{2}\left[\mu \lambda \cos \left(\varphi_{1}\right)+\cos \left(\varphi_{2}\right)\right]\right\} .
\end{aligned}
$$

Before we proceed, let us comment on certain trivial symmetries of the quantity $E$. Firstly, eq. (20) is invariant under $\omega_{i} \rightarrow-\omega_{i}$. Since this is not interesting physically, we add the restriction $\omega_{1} \geqslant 0$ to the already existing ones. Secondly, the factor of $m_{2} l_{1} l_{2}$ in front of the curly bracket in (20) indicates that this term cannot be determined by our approach because the solution of the minimization is only determined up to a multiplicative constant (unless measured values of energy are given externally). However, this factor does not show up in the differential equations either - which is to say that $\lambda, \mu$, and $\omega_{1}$ are the only relevant parameters of the problem. This is again related to the arbitrariness in choosing the units of measurement.

For the integration of our model trajectory, we chose the parameters $\lambda=1, \mu=2$, and $\omega_{1}=\sqrt{9.81} \approx 3.132$. The data points were then sampled from the initial values $\left(\dot{\varphi}_{1}, \dot{\varphi}_{2}, \varphi_{1}, \varphi_{2}\right)_{0}=\left(\omega_{1}, \omega_{1}, \frac{1}{2} \pi, \frac{1}{2} \pi\right)$ with equidistant time steps of $\Delta t=0.25$ (this yields about ten to twelve data points per oscillation). The total energy is nearly half the difference of the maximal and minimal potential energy (i.e., of $V$ at $\varphi_{1}=\varphi_{2}=\pi$ and $V$ at $\varphi_{1}=\varphi_{2}=0$ ) where the system is known to behave chaotically [19]. The situation with ideal data was very much the same as above, i.e., parameters were found with at least six digits accuracy - without dependence on initial guesses, number of points $(\geqslant 10)$, and their selection from the orbit.

As above, a considerable amount of noise could be treated. With $50(100)$ points used for the fit, Gaussian noise of $5 \%(10 \%)$ standard deviation (see above, angles were taken $\bmod 2 \pi$ before averaging) resulted in errors of some $5 \%(13 \%)$ for $\mu$ versus $2 \%(6 \%)$ for $\lambda$ and $\omega_{1}$. In view of this robustness (we have nonlinear dependence on the parameters, but only three of them), it is not too astonishing that the reconstruction of angular velocities by numerical differentiation (fourth order formula) did not spoil parameter estimation: with 50 points, errors stayed below $5 \%$, and even the reconstruction from noisy data (5\% Gaussian noise, 100 points) resulted in errors around $10 \%$ for $\lambda, \omega_{1}$ and $30 \%$ for $\mu$.

We chose the double pendulum with its nonlinear parameter dependence in order to also test the performance of the surface fitting approach (4). To solve the constrained least squares problem, we adopted the subroutine E04UPF from the NAG library (a sequential quadratic programming algorithm). For complete phase space information, we obtained slightly more precise results, at the cost of a smaller radius of convergence concerning the initial estimates for the parameters. For noise and/or incomplete data, precision increase was more significant (relative errors were roughly halved) wherefrom we conclude that the full surface fitting approach is superior in this situation. Let us now turn to our final example of a discrete dynamical system.

\section{The Fibonacci trace map}

Although the technique described above is most advantageous for continuous dynamical systems because one shortcuts the effort of numerical integration, it is obviously not restricted to this case. On the contrary, one can apply it to discrete systems with invariants as well and one gets a simple approach to global fitting (for local fitting, we refer to ref. [13] and references therein). Let us explain this by means of the Fibonacci trace map [20]

$x_{n+1}=2 x_{n} x_{n-1}-x_{n-2}$, 
which is a discrete iteration of recursion depth 3 . As such, $(21)$ is a $3 \mathrm{D}$ discrete dynamical system with various physical applications, compare ref. [21] and references therein, and can be derived from a continuous dynamical system without approximation [22].

This system is reversible [23] and possesses the invariant $[20,24]$

$\tilde{I}\left(x_{n-1}, x_{n}, x_{n+1}\right)=x_{n-1}^{2}+x_{n}^{2}+x_{n+1}^{2}-2 x_{n-1} x_{n} x_{n+1}-1$.

For details on the structure we refer to ref. [25] and references therein. If $I=0$ and $\left|x_{i}\right| \leqslant 1, i=1,2,3$, we are in a region of homogeneous chaos. This follows from a semi-conjugacy to a hyperbolic automorphism of the torus which makes the system pseudo-Anosov $[25,16]$,

$x_{n}=\cos \left(2 \pi \vartheta_{n}\right), \quad \vartheta_{n+1}=\vartheta_{n}+\vartheta_{n-1}$.

We will take test orbits from this region where the calculation has been done with quadruple precision arithmetic. The latter, when calculated by (23), gives reliable orbits of length $>100$ which is sufficient for our purposes.

Now, we have to specify our model class. The more general iteration

$x_{n+1}=a+b\left(x_{n}+x_{n-1}\right)+c x_{n} x_{n-1}-x_{n-2}$

still has an invariant, namely [25]

$$
\begin{gathered}
I\left(x_{n-1}, x_{n}, x_{n+1}\right)=x_{n-1}^{2}+x_{n}^{2}+x_{n+1}^{2}-a\left(x_{n-1}+x_{n}+x_{n+1}\right) \\
-b\left(x_{n-1} x_{n}+x_{n-1} x_{n+1}+x_{n} x_{n+1}\right)-c x_{n-1} x_{n} x_{n+1},
\end{gathered}
$$

which can be easily be checked by direct substitution. We have dropped a constant contribution in comparison with (22) for convenience.

The task now is to identify the original Fibonacci system out of this three-parameter class of dynamical systems. Again, we used the simple Monte Carlo program mentioned above. The result is, very briefly, that $c=2$, $a=b=0$ was always found with at least seven digits precision for ideal data $(10,20$, or 50 points, systematic or random). With a sample of 50 points, noise of $1 \%(10 \%)$ standard deviation (see above) resulted in an error of $0.2 \%(2 \%)$ for $c$ while $|a|,|b|$ were below $5 \times 10^{-4}\left(1 \times 10^{-2}\right)$ and $F_{3}$ was around $10^{-4}\left(10^{-2}\right)$. This situation is even more robust than those of the previous sections but one could expect that because of the relatively simple structure of (24). In any case, the treatment of chaotic orbits, especially in the presence of noise, seems to be more robust than that of non-chaotic ones (periodic, say) because the former typically explore a larger portion of state space.

\section{Concluding remarks}

We have demonstrated by means of three examples (taken from two continuous and one discrete dynamical system ) that the parameter estimation problem in the presence of an invariant is facilitated considerably if the parameters to be determined show up in the invariant. We have indicated that one can tackle noise as well as arbitrarily low sampling rates where methods relying on numerical integration catch problems in the chaotic case. Even incomplete data are tractable if the sampling rate is high enough to allow numerical estimates of missing components. The latter problem, however, is probably better treated with multiple shooting algorithms $[1,2]$, but from the simpler approach with the invariant one could obtain a reasonable start estimate. Therefore, it might be useful to combine both methods.

Let us close with a remark on another application of the method presented. If, for a given dynamical system, one wants to known whether it has an invariant, one can treat that quite easily for discrete systems by means of algebraic program packages. For continuous systems, however, the corresponding analysis is much more 
elaborate (see ref. [27] for a review). In searching for an invariant in a certain class of functions, one could try a numerical simulation based on eq. (7) as a first and easy go. At least in the case that the minimization converges, one obtains a good guess for an invariant to be tested analytically.

\section{Acknowledgement}

It is a pleasure to thank K.M. Briggs for various discussions, S.I. Ben-Abraham and J.A.G. Roberts for critically reading the manuscript, and the referee for helpful and constructive comments. This work was supported by the Deutsche Forschungsgemeinschaft.

\section{References}

[1] E. Baake, M. Baake, H.G. Bock and K.M. Briggs, Phys. Rev. A 45 (1992) 5524.

[2] J. Kallrath, H.G. Bock and J.P. Schlöder, Heidelberg preprint 92-29.

[3] H.G. Bock, in: Modelling of chemical reaction systems, Springer series in chemical physics, eds. K.H. Ebert, P. Deuflhard and W. Jäger (Springer, Berlin, 1981); in: Bonner Mathematische Schriften, Band 183, eds. E. Brieskorn et al. (Bonn, 1987).

[4] P.E. Gill, W. Murray and M.H. Wright, Practical optimization (Academic Press, New York, 1981 ).

[5] E. Eich, SIAM J. Numer Anal. 30 (1993), in press.

[6] T.S. Parker and L.O. Chua, Practical numerical algorithms for chaotic systems (Springer, Berlin, 1989).

[7] L.F. Shampine, Comput. Math. Appl. Ser. A 12 (1986) 885.

[8] C.W. Gear, SIAM J. Sci. Stat. Comput. 7 (1986) 734.

[9] E. Eich, Thesis, Universität Augsburg (1991); VDI-Fortschrittsberichte, Reihe 18, Nr. 109 (1992).

[10] R.I. McLachlan and P. Atela, Nonlinearity 5 (1992) 541;

E. Forest, J. Comput. Phys. 99 (1992) 209.

[11] Z. Ge and J.E. Marsden, Phys. Lett. A 133 (1988) 134; R.S. MacKay, Inst. Math. Appl. Conf. Ser. New Ser. 34 (1992) 137.

[12] S. Miesbach and H.J. Pesch, Numer. Math. 61 (1992) 501; K.M. Briggs, private communication (1992).

[13] K.M. Briggs, Phys. Lett. A 151 (1990) 27.

[14] M. Hénon and C. Heiles, Astronom. J. 69 (1964) 16.

[15] J.K. Moser, Mem. Am. Math. Soc. 81 (1968) 1; M.V. Berry, Am. Inst. Phys. Conf. Proc. 46 (1978) 16; both reprinted in: Hamiltonian dynamical systems, eds. R.S. MacKay and J.D. Meiss (Hilger, Bristol, 1987).

[16] W.H. Press, B.P. Flannery, S.A. Teukolsky and W.T. Vetterling, Numerical recipes (Cambridge Univ. Press, Cambridge, 1989).

[17] G.H. Golub and C.F. van Loan, Matrix computations, 2nd Ed. (Johns Hopkins Univ. Press, Baltimore, 1989).

[ 18 ] L.D. Landau and E.M. Lifschitz, Mechanik, 11 th Ed. (Akademie-Verlag, Berlin, 1984).

[19] P.H. Richter and H.-J. Scholz, The planar double pendulum, Film C1574, Naturw. Ser. 9, Nr. 7/C1574, Publ. Wiss. Film, Sekt. Techn. Wiss., Göttingen (1986).

[20] M. Kohmoto, L.P. Kadanoff and C. Tang, Phys. Rev. Lett. 50 (1983) 1870; S. Ostlund, R. Pandit, D. Rand, H.-J. Schellnhuber and E.D. Siggia, Phys. Rev. Lett. 50 (1983) 1873; both (and several related ones) reprinted in: The physics of quasicrystals, eds. P.J. Steinhardt and S. Ostlund (World Scientific, Singapore, 1987).

[21] M. Baake, D. Joseph and P. Kramer, Phys. Lett. A 168 (1992) 199;

M. Baake, U. Grimm and D. Joseph, Int. J. Mod. Phys. B 7 (1993) 1527.

[22] P. Kramer, J. Phys. A 26 (1993) 213.

[23] J.A.G. Roberts and G.R.W. Quispel, Phys. Rep. 216 (1992) 63.

[24] R. Fricke and F. Klein, Vorlesungen über die Theorie der automorphen Funktionen, Band 1 (Teubner, Leipzig, 1897).

[25] J.A.G. Roberts and M. Baake, Melbourne preprint 9-1992, submitted to J. Stat. Phys.

[26] R.L. Devaney, An introduction to chaotic dynamical systems, 2nd Ed (Addison-Wesley, Reading, MA, 1989).

[27] J. Hieterinta, Phys. Rep. 147 (1987) 87. 